\title{
Prognostic significance of histone methyltransferase enhancer of zeste homolog 2 in patients with cervical squamous cell carcinoma
}

\author{
MINFEI JIN ${ }^{1 *}$, ZUJING YANG $^{1 *}$, WEIPING YE ${ }^{1}$, XIAOWEI YU ${ }^{2}$ and XIAOLIN HUA ${ }^{1}$ \\ ${ }^{1}$ Department of Obstetrics and Gynecology, Xinhua Hospital, Shanghai Jiao Tong University School of Medicine, \\ Shanghai 200092; ${ }^{2}$ Department of Orthopedics, Shanghai Sixth People's Hospital, \\ Shanghai Jiao Tong University, Shanghai 220023, P.R. China
}

Received April 13,2014; Accepted January 13, 2015

DOI: $10.3892 / 01.2015 .3319$

\begin{abstract}
Histone methyltransferase enhancer of zeste homolog $2(\mathrm{EZH} 2)$ has been reported to be associated with certain malignant phenotypes in cervical cancer. However, clinicopathological parameters and clinical outcomes of EZH2 in cervical cancer, particularly in cervical squamous cell carcinoma (CSCC) remain largely unknown. The retrospective cohort comprising of 117 consecutive patients with CSCC was incorporated into a tissue microarray which also included 23 paired normal tissues. Immunohistochemical analysis was performed to evaluate the correlation between EZH2 expression and clinicopathological implications. Aberrant overexpression of EZH2 was frequently observed in CSCCs as compared with adjacent normal tissues $(\mathrm{P}=0.0005)$. Expression of EZH2 is associated with poor tumor differentiation grade $(\mathrm{P}=0.020)$ and lymphovascular invasion $(\mathrm{P}=0.012)$. Univariate analysis revealed that the patients with CSCC whose tumors exhibited higher EZH2 levels had inferior overall survival (OS) compared to those whose tumors expressed lower EZH2 ( $\log$ rank $\mathrm{P}=0.004)$. In the multivariate analysis, EZH2 expression was an independent predictor of OS (hazard ratio $=1.836$, 95\% confidence interval: $1.090-2.993, \mathrm{P}=0.022)$. EZH2 overexpression is common in the development of CSCC and is a promising prognostic predictor for patients with CSCC.
\end{abstract}

Correspondence to: Dr Xiaolin Hua, Department of Obstetrics and Gynecology, Xinhua Hospital, Shanghai Jiao Tong University School of Medicine, 1665 Kongjiang Road, Shanghai 200092, P.R. China

E-mail: huaxiaolin_sh@163.com

${ }^{*}$ Contributed equally

Abbreviations: CSCC, cervical squamous cell carcinoma; EZH2, enhancer of zeste homolog 2; OS, overall survival; FIGO, International Federation of Gynecology and Obstetrics

Key words: histone methyltransferase, EZH2, immunohistochemistry, cervix, prognosis

\section{Introduction}

Cervical cancer is the third most common type of cancer in females, accounting for $9.8 \%$ of all new cancer cases worldwide (1). Squamous cell carcinoma (SCC) is the most common type of cervical cancer, accounting for $80-90 \%$ (2). Through cancer screening using the Papanicolaou test, there has been improved survival in patients with early-stage cervical cancer; however, advanced stages of this disease treated with surgery, chemotherapy and/or radiotherapy are not significantly altered (3). This mostly likely is due to the absence of a well-established understanding of the molecular basis of cervical SCC (CSCC) progression. Furthermore, consolidated prognostic or therapeutic targets for CSCC in clinical practice are urgently required.

Epigenetic modifications to inactivate tumor suppressor genes are a common molecular mechanism involved in tumor initiation and progression (4). Enhancer of zeste homolog 2 (EZH2) is the main part of the polycomb repressor complex (PRC) 2, which possesses histone methyltransferase activity, mediating histone $\mathrm{H} 3$ at lysine 27 trimethylation (H3K27Me3) (5). It has been demonstrated that $\mathrm{EZH} 2$ is directly involved in the silencing of a subgroup of genes implicated in cell differentiation by the use of its histone methyltransferase activity (6). Highly conserved motifs pThr350 and pThr487 localized in EZH2 were identified as important for EZH2 recruitment and H3K27Me3 level maintenance $(7,8)$. Additionally, it was also shown that EZH2 as a landmark can recruit DNA methyltransferases and indirectly induce DNA methylation $(9,10)$. Aberrant overexpression of EZH2 was observed in multiple solid tumor types and precancerous lesions, and was associated with a poor prognosis in patients with those types of cancer (11-18). However, EZH2 has been proven to be composed of distinct biological functions in a tissue-specific manner in certain tumors since it is associated with improved prognosis in those diseases $(19,20)$. Although biological roles of EZH2 were shown to be involved in cervical cancer cell proliferation and invasion by virus and oncoprotein activation, the prognostic significance of EZH2 in cervical cancer remains unclear. The present study sought to determine the clinical implications of $\mathrm{EZH} 2$ overexpression in CSCC and to explore the possibility of EZH2 as a prognostic predictor for patients with CSCC. 
Table I. Associations between EZH2 protein levels and clinical parameters in patients with cervical squamous cell carcinoma $(\mathrm{n}=117)$.

\begin{tabular}{|c|c|c|c|}
\hline \multirow[b]{2}{*}{ Characteristic } & \multicolumn{2}{|c|}{ EZH2 expression } & \multirow[b]{2}{*}{ P-value } \\
\hline & $<$ Median & $\geq$ Median & \\
\hline Age, years & & & 0.476 \\
\hline Mean \pm standard deviation & $57.9 \pm 12.1$ & $55 \pm 12.9$ & \\
\hline Median & 58 & 55 & \\
\hline Range & $26-83$ & $21-85$ & \\
\hline FIGO stage & & & 0.100 \\
\hline IB1, n (\%) & $38(32.5)$ & $47(40.2)$ & \\
\hline$>\mathrm{IB} 1, \mathrm{n}(\%)$ & $20(17.1)$ & $12(10.3)$ & \\
\hline Tumor size & & & 0.849 \\
\hline$<4 \mathrm{~cm}, \mathrm{n}(\%)$ & $28(23.9)$ & $28(23.9)$ & \\
\hline$\geq 4 \mathrm{~cm}, \mathrm{n}(\%)$ & $30(25.6)$ & $31(26.5)$ & \\
\hline Lymphovascular invasion & & & 0.012 \\
\hline Negative, n (\%) & $30(25.6)$ & $18(15.4)$ & \\
\hline Positive, n (\%) & $24(20.5)$ & $40(34.2)$ & \\
\hline Missing, n (\%) & $4(3.4)$ & $1(0.9)$ & \\
\hline Tumor differentiation & & & 0.020 \\
\hline Well, n (\%) & $51(43.6)$ & $41(35.0)$ & \\
\hline Moderate, $\mathrm{n}(\%)$ & $7(6.0)$ & $15(12.8)$ & \\
\hline Poor, n (\%) & $0(0.0)$ & $3(2.6)$ & \\
\hline Lymph node metastasis & & & 0.564 \\
\hline Negative, n (\%) & $39(33.3)$ & $36(30.8)$ & \\
\hline Positive, n (\%) & $19(16.2)$ & $23(19.7)$ & \\
\hline
\end{tabular}

FIGO, International Federation of Gynecology and Obstetrics; EZH2, enhancer of zeste homolog 2.

\section{Materials and methods}

Patients and specimens. The present study was reviewed and approved by the institutional ethical committee of Xinhua Hospital (Shanghai, China). All subjects signed informed consent documents for participation in this study. A total of 117 consecutive patients were assembled from the database based on the histological diagnosis of primary CSCC who received radical surgery in the Department of Obstetrics and Gynecology, Xinhua Hospital (Shanghai, China) between January 2000 and August 2005. Exclusion criteria included recurrent or distant metastasis at presentation, preoperative radiotherapy, chemotherapy and incomplete medical records. The medical records of patients with CSCC were retrospectively reviewed according to the inclusion and exclusion criteria. Clinicopathological parameters of patients with CSCC are summarized in Table I. The median follow-up of the patients was seven years. In the current study, tumors from each patient were stained with hematoxylin and eosin (H\&E), classified histologically by three experienced pathologists, and clinically staged according to the International Federation of Gynecology and Obstetrics (FIGO) criteria (21) prior to further analysis as follows: 85 were allocated to stage IB1, 10 to stage IB2, eight to stage IIA1, eight to stage IIA2 and six to stage IIB. The median age of the patients was 56.5 years.
Tissue microarray (TMA) construction and immunohistochemistry (IHC). For TMA construction, slides were reviewed by a pathologist and areas containing each category were annotated on the H\&E slides (Sangon Biotech Corp., Shanghai, China) Three cylindrical tissue cores of $1.0 \mathrm{~mm}$ diameter from each patient were taken from the corresponding regions of the paraffin blocks (Sangon Biotech Corp.) and transplanted into a recipient paraffin block. For IHC, paraffin block-embedded tissue specimens were cut into $4 \mu \mathrm{m}$ tissue sections. Briefly, tissue sections were deparaffinized in xylene (Sangon Biotech Corp.), rehydrated in graded ethanol (Sangon Biotech Corp.), treated with Tris-ethylene diamine tetraacetic acid buffer (Sigma-Alrich, St. Louis, MO, USA) for antigen retrieval and quenched in hydrogen peroxide (Sigma-Aldrich). Tissue sections were blocked with $2.5 \%$ normal goat serum (Epitomics, Inc., Shanghai, China) incubated at $4^{\circ} \mathrm{C}$ overnight with a monoclonal mouse anti-human EZH2 antibody (1:200 dilution; clone 11; BD Transduction Laboratories, San Jose, CA, USA) followed by incubation with a monoclonal goat anti-mouse IgG secondary antibody (1:500 dilution; cat. no. SA00001-1; Proteintech Corp., Shanghai,China). Diaminobenzidine was used as the chromogen, and sections were counterstained with Mayer's hematoxylin (Sigma-Aldrich) and visualized using a microscope (TE300; Nikon Corporation, Tokyo, Japan). The immunoreactive score was defined semi-quantitatively as the intensity of staining $(0,1$, 
Table II. Univariate and multivariate cox proportional hazard regression models in estimating overall survival.

\begin{tabular}{lcc}
\hline Characteristics & HR & 95\% CI \\
\hline Univariate analysis & & $0.539-1.585$ \\
Age ( $\geq 60$ vs. $<60$ ) & 0.925 & $1.186-5.244$ \\
FIGO stage (IB1 vs. >IB1) & 2.493 & $0.243-1.072$ \\
Tumor size (<4 cm vs. $\geq 4$ cm) & 0.510 & $1.060-2.453$ \\
Tumor differentiation & 1.612 & $0.909-3.061$ \\
Lymphovascular invasion (negative vs. positive) & 1.838 & $1.332-5.264$ \\
Lymph node metastasis (negative vs. positive) & 2.648 & $1.366-6.738$ \\
EZH2 expression (high vs. low) & 3.034 & 0.076 \\
Multivariate analysis & & 0.008 \\
FIGO stage (IB1 vs. >IB1) & 1.341 & 0.005 \\
Tumor differentiation & 1.328 & 0.006 \\
EZH2 expression (high vs. low) & 1.836 & $0.825-2.139$ \\
Lymphovascular invasion (negative vs. positive) & 1.868 & $1.090-2.993$ \\
Lymph node metastasis (negative vs. positive) & 2.071 & $1.039-3.222$ \\
\hline
\end{tabular}

FIGO, International Federation of Gynecology and Obstetrics; HR, hazard ratio; CI, confidence interval; EZH2, enhancer of zeste homolog 2.

A

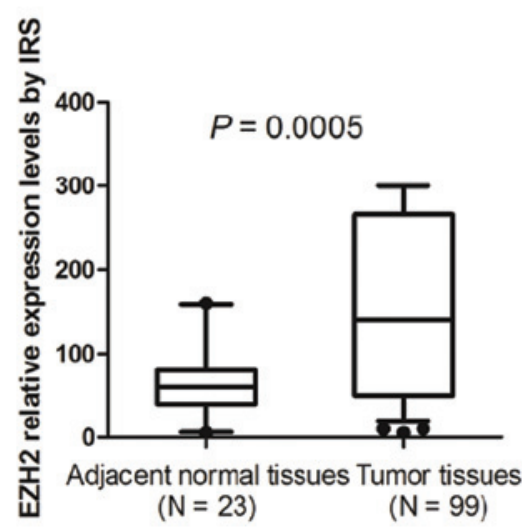

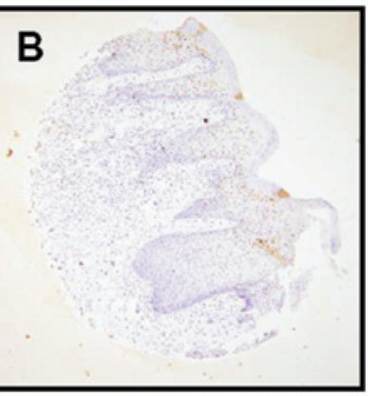
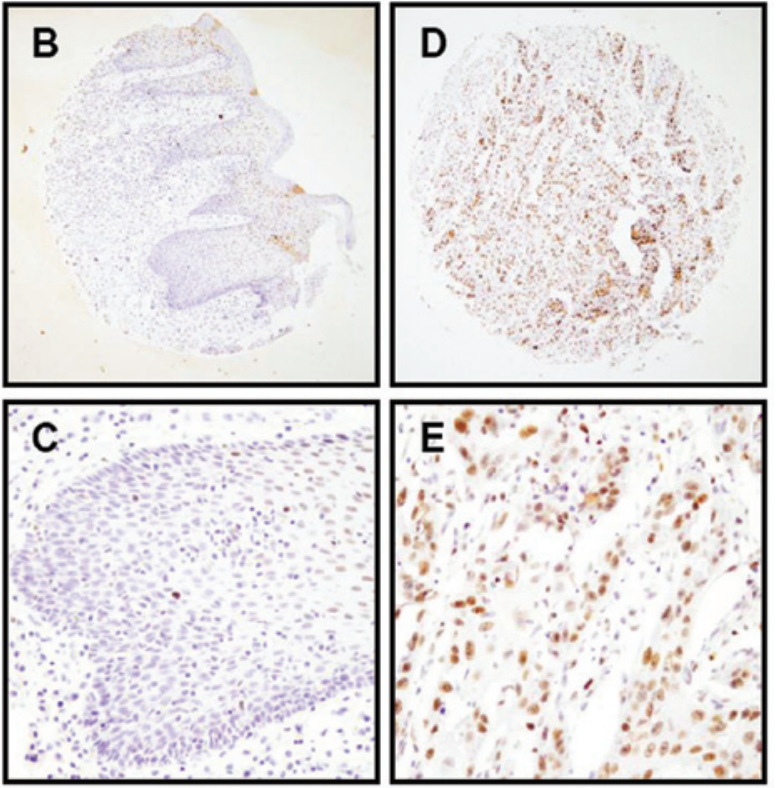

Figure 1. Expression patterns of enhancer of zeste homolog 2 (EZH2) were served in 117 cervical squamous cell carcinomas (CSCCs) and 23 adjacent normal tissues. (A) Histogram shows expression levels of EZH2 in CSCC and adjacent normal tissues by immunoreactive score (IRS). (B and C) Expression and localization of EZH2 in adjacent normal tissues (A, original magnification, x100; B, original magnification, x400). (D and E) Expression and localization of EZH2 in CSCCs (C, original magnification, x100; D, original magnification, $\mathrm{x} 400)$.

2 or 3) multiplied by the percentage of positive tumor cells with nuclear staining $(25,50,75$ or $100 \%)$. Two independent pathologists randomly examined five tumor cell areas in each dot of the microarray, and a cutoff value was set as the median of the immunoreactive score. For EZH2 staining, the cutoff value was 120 ; thus, a value $\geq 120$ was considered high expression, and a value $<120$ was considered low expression.

Statistical analysis. Frequency distributions were used to summarize categorical data, and medians, interquartile ranges and ranges were used to summarize continuous data.
The associations between EZH2 expression levels and patient characteristics were evaluated using Fisher's exact tests for categorical variables. Probabilities of overall survival were estimated using the log-rank test. All potential prognostic factors with $\mathrm{P}<0.05$ from the univariate analysis were incorporated into the multivariate analysis. The hazard ratios with corresponding $95 \%$ confidence interval (CI) and P-value were exported. All tests were two-sided, and $\mathrm{P}<0.05$ was considered to indicate a statistically significant difference. All analyses were conducted using the SPSS software program, standard version 13.0 (SPSS, Inc., Chicago, IL, USA). 


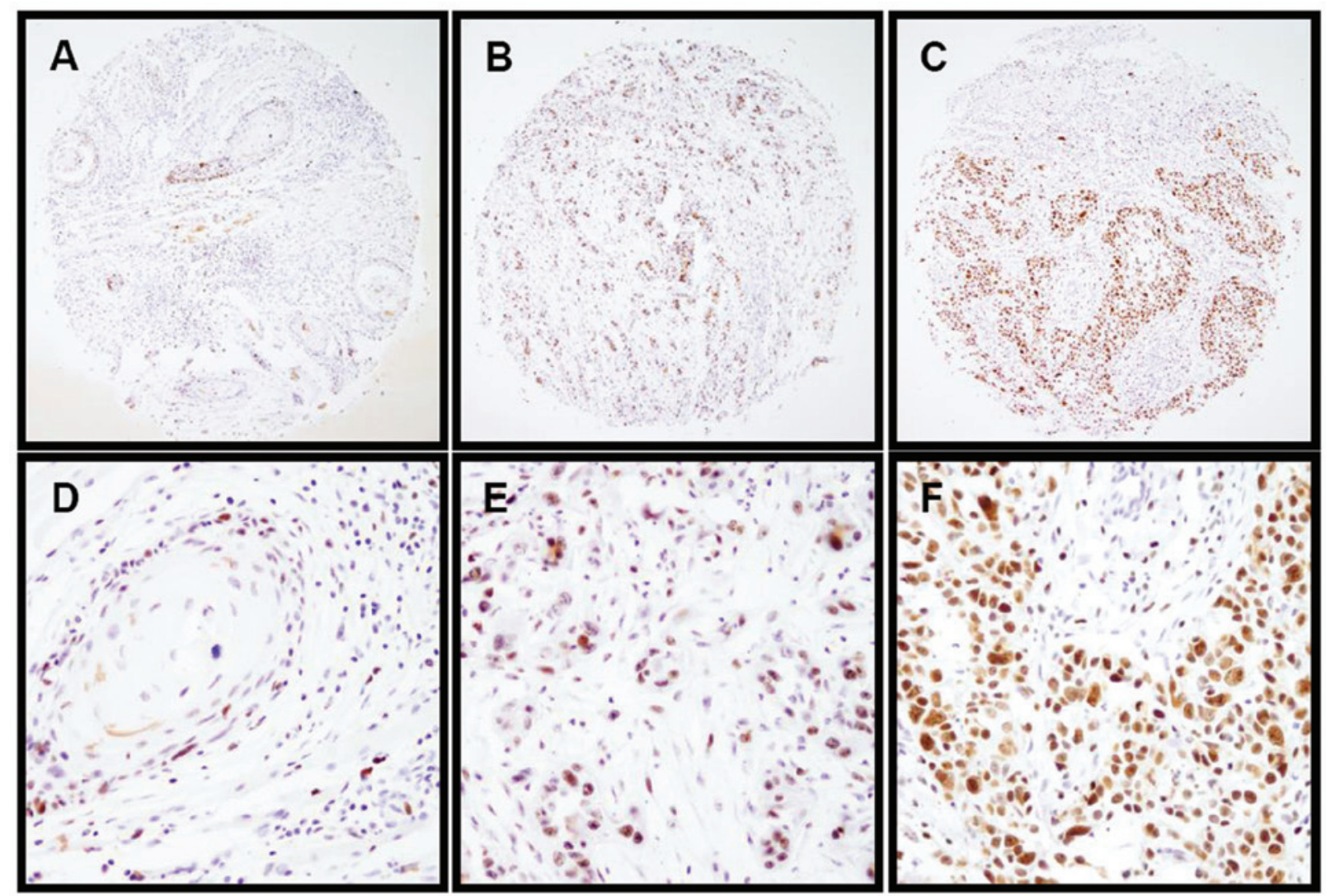

Figure 2. Patterns of EZH2 expression were investigated in CSCC specimens. Representative images show immunohistochemical staining for EZH2 expression in (A) well-differentiated CSCCs, (B) moderately differentiated CSCCs and (C) poorly differentiated CSCCs (original magnification, x100). (D-F) Corresponding amplified images for (A-C) (original magnification, x400). EZH2, enhancer of zeste homolog 2; CSCC, cervical squamous cell carcinoma.

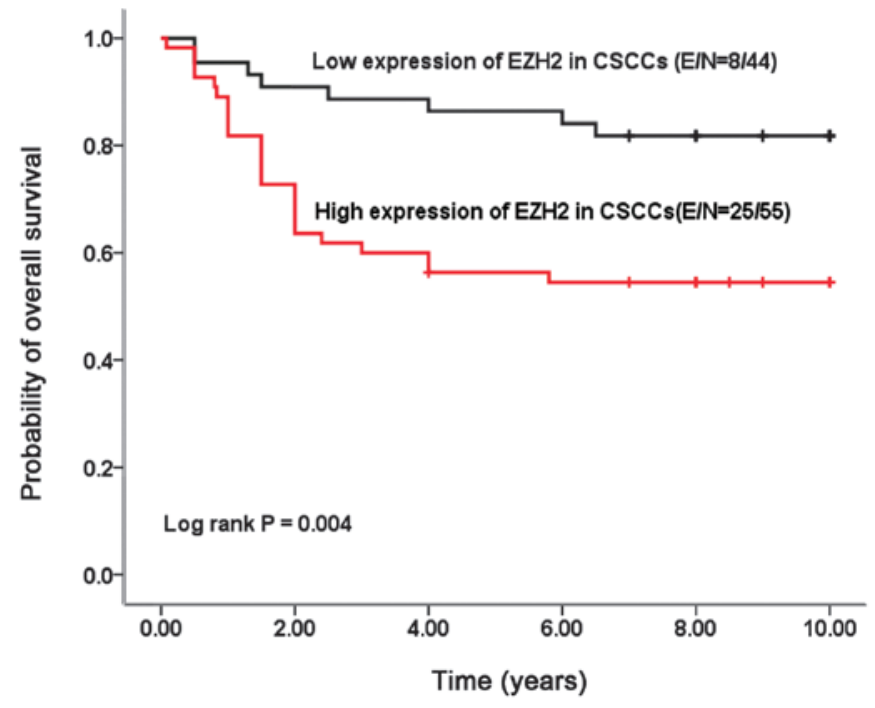

Figure 3. The Kaplan-Meier survival curve illustrates overall survival according to nuclear staining for EZH2. EZH2, enhancer of zeste homolog 2; CSCC, cervical squamous cell carcinoma

\section{Results}

Overexpression of EZH2 was observed in patients with CSCC. The tissue microarray in this study includes 117 CSCC samples and 23 paired adjacent normal tissues. Immunohistochemical results showed that aberrantly upregulated EZH2 was commonly observed in CSCC tissues compared with paired adjacent normal tissues ( $\mathrm{P}=0.0005)$ (Fig. 1A). Of 23 paired adjacent normal tissues, EZH2 was mainly found to be localized in the epithelial layer, whereas EZH2 expression was not notably detected in the basal cell layer (Fig. 1B-E).

Expression of EZH2 is associated with CSCC clinicalfeatures. To further investigate the clinical implications of EZH2 in CSCCs, the correlation between EZH2 expression and the clinical characteristics in 117 CSCC cases was examined. The present data demonstrated that high expression of EZH2 was frequently detected in poorly differentiated tumors $(\mathrm{P}=0.020)$ (Fig. 2) and patients with lymphovascular invasion $(\mathrm{P}=0.012)$. No significant associations were identified between EZH2 expression pattern and age $(\mathrm{P}=0.476)$, tumor size $(\mathrm{P}=0.849)$, FIGO stage $(\mathrm{P}=0.100)$ or lymph node metastasis $(\mathrm{P}=0.564)$ (Table I).

Association between EZH2 expression and clinical outcome in patients with CSCC. To determine the correlation between EZH2 expression and clinical prognosis, overall survival probability was estimated using Kaplan-Meier curve analysis. The present findings showed that patients whose primary tumors possessed a high level of EZH2 (the median of the immunoreactive score of 120 was used as a cutoff point) had significantly decreased overall survival rates $(\mathrm{P}=0.004)$ 
(Fig. 3). In the univariate and multivariate Cox proportional analyses (Table II), EZH2 expression status (hazard ratio, 1.836; 95\% CI, 1.090-2.993; $\mathrm{P}=0.022$ ), together with lymphovascular invasion (hazard ratio, 1.868; 95\% CI, 1.039-3.222; $\mathrm{P}=0.014$ ) and lymph node metastasis (hazard ratio, 2.071; 95\% CI, 1.186-3.617; $\mathrm{P}=0.010)$, is identified as an independent predictor of overall survival time in patients with CSCC.

\section{Discussion}

In addition to genetic alterations, epigenetic modifications were shown to be the one of the key molecular mechanisms involved in tumor initiation and development (22). Previous findings showed that EZH2, one of the histone methyltransferases, plays a vital role in cell differentiation and maintenance of stem cell self-renewal by catalyzing H3-K27 trimethylation, leading to transcriptional inactivation of target genes (23). Furthermore, upregulation of EZH2 has been observed in several human epithelial cancer types including gynecologic cancer $(24,25)$. Considering the particularity of CSCC epidemiology, which had the distinct molecular signatures involved in initiation and progression, the expression pattern of EZH2 in 117 CSCC samples and 23 paired adjacent normal tissues was evaluated. Consistent with previous results (26), aberrant EZH2 overexpression in CSCC indicates it is a common event in the tumor type (Fig. 1) $(\mathrm{P}=0.0005)$.

In a prior in vitro study, blocking EZH2 gene expression using RNA interference resulted in the inhibition of cell proliferation, cell cycle arrest and invasion in cervical cancer cells by upregulation of p21 and downregulation of proliferating cell nuclear antigen, suggesting its biological function in maintaining the malignant behaviors of cervical cancer (27). Additionally, an in vivo study revealed that high EZH2 levels were significantly associated with differentiation, depth and lymphatic metastasis of cervical cancers (27). However, the clinical implication of EZH2 expression in CSCC, which accounts for almost $90 \%$ of cervical cancers, remains unrevealed. In the current study, to investigate the clinical correlations, expression of EZH2 levels were evaluated in 117 patients with CSCC. The present results showed that EZH2 expression patterns were notably associated with tumor grade (Fig. 2) and lymphovascular invasion (Table I).

A key aim of studying molecular abnormalities in cancers is to be translated into clinical applications. The limitations of conventional treatments in CSCC have necessitated a search for novel potential tools. The driving molecular targets could provide novel insights for early diagnosis, prognosis and new personal targeted therapies. In this study, immunohistochemical staining was applied to determine EZH2 expression status in a cohort of patients with CSCC and the association of EZH2 expression and clinical outcome of these patients. EZH2 staining was found exclusively in nuclei as shown in previous studies $(13,28)$. Furthermore, for the first time, these findings showed that the aberrant overexpression of EZH2 correlates with the poor survival rates of patients with CSCC (Fig. 3). Univariate and multivariate analyses demonstrated that EZH2 expression level (hazard ratio, 1.836; 95\% CI, 1.090-2.993; $\mathrm{P}=0.022$ ), together with lymphovascular invasion (hazard ratio, 1.868; 95\% CI,
1.039-3.222; $\mathrm{P}=0.014)$ and lymph node metastasis (hazard ratio, 2.071; 95\% $\mathrm{CI}, 1.186-.617 ; \mathrm{P}=0.010)$, are independent prognosticators for patients with CSCC (Table II). Together with other biomarkers, EZH2 status may aid the implementation of optimized therapeutic strategies for CSCC. Taken together, in the current study, IHC was used to establish the association between aberrant EZH2 expression and malignant phenotypes of CSCC. Furthermore, the results demonstrate that $\mathrm{EZH} 2$ expression status is an independent predictor of clinical outcome in patients with CSCC.

\section{Acknowledgements}

The authors gratefully acknowledge the foundation supported by the National Natural Science Foundation of China (no. 81401186); the Natural Science Foundation of Shanghai Municipal Health Bureau (no. 20114y079); the Medicine and Engineering Foundation of Shanghai Jiao tong University (no. YG2011MS33); the Natural Science Foundation of Xinhua Hospital (no. 09QYJ05); the Fifth Special China Postdoctoral Science Foundation (no. 2012T50420); the Program for Medical Talents of Jiangsu Province (no. H201122) and the High-Level Talents of Six Major Industries of Jiangsu Province (no. WSN-042).

\section{References}

1. Vizcaino AP, Moreno V, Bosch FX, et al: International trends in incidence of cervical cancer: II. Squamous-cell carcinoma. Int J Cancer 86: 429-435, 2000.

2. Koutsky L: Epidemiology of genital human papillomavirus infection. Am J Med 102: 3-8, 1997.

3. Moore DH, Blessing JA, McQuellon RP, et al: Phase III study of cisplatin with or without paclitaxel in stage IVB, recurrent, or persistent squamous cell carcinoma of the cervix: a gynecologic oncology group study. J Clin Oncol 22: 3113-3119, 2004.

4. Baylin SB and Ohm JE: Epigenetic gene silencing in cancer-a mechanism for early oncogenic pathway addiction? Nat Rev Cancer 6: 107-116, 2006.

5. Simon JA: Transcription. Sweet silencing. Science 325: 45-46, 2009.

6. Valk-Lingbeek ME, Bruggeman SW and van Lohuizen M: Stem cells and cancer; the polycomb connection. Cell 118: 409-418, 2004.

7. Wei Y, Chen YH, Li LY, et al: CDK1-dependent phosphorylation of $\mathrm{EZH} 2$ suppresses methylation of $\mathrm{H} 3 \mathrm{~K} 27$ and promotes osteogenic differentiation of human mesenchymal stem cells. Nat Cell Biol 13: 87-94, 2011.

8. Chen S, Bohrer LR, Rai AN, et al: Cyclin-dependent kinases regulate epigenetic gene silencing through phosphorylation of EZH2. Nat Cell Biol 12: 1108-1114, 2010.

9. Viré E, Brenner C, Deplus R, et al: The Polycomb group protein EZH2 directly controls DNA methylation. Nature 439: 871-874, 2006.

10. Gieni RS and Hendzel MJ: Polycomb group protein gene silencing, non-coding RNA, stem cells, and cancer. Biochem Cell Biol 87: 711-746, 2009.

11. Varambally S, Dhanasekaran SM, Zhou M, et al: The polycomb group protein $\mathrm{EZH} 2$ is involved in progression of prostate cancer. Nature 419: 624-629, 2002.

12. Gong Y, Huo L, Liu P, et al: Polycomb group protein EZH2 is frequently expressed in inflammatory breast cancer and is predictive of worse clinical outcome. Cancer 117: 5476-5484, 2011.

13. Cao W, Feng Z, Cui Z, et al: Up-regulation of enhancer of zeste homolog 2 is associated positively with cyclin D1 overexpression and poor clinical outcome in head and neck squamous cell carcinoma. Cancer 118: 2858-2871, 2012.

14. Cao W, Younis RH, Li J, et al: EZH2 promotes malignant phenotypes and is a predictor of oral cancer development in patients with oral leukoplakia. Cancer Prev Res (Phila) 4: 1816-1824, 2011.

15. Cao W, Ribeiro Rde O, Liu D, et al: EZH2 promotes malignant behaviors via cell cycle dysregulation and its mRNA level associates with prognosis of patient with non-small cell lung cancer. PLoS One 7: e52984, 2012. 
16. Kikuchi J, Kinoshita I, Shimizu Y, et al: Distinctive expression of the polycomb group proteins Bmil polycomb ring finger oncogene and enhancer of zeste homolog 2 in nonsmall cell lung cancers and their clinical and clinicopathologic significance. Cancer 116: 3015-3024, 2010

17. Huqun, Ishikawa R, Zhang J, et al: Enhancer of zeste homolog 2 is a novel prognostic biomarker in nonsmall cell lung cancer. Cancer 118: 1599-1606, 2012

18. He LR, Liu MZ, Li BK, et al: High expression of EZH2 is associated with tumor aggressiveness and poor prognosis in patients with esophageal squamous cell carcinoma treated with definitive chemoradiotherapy. Int J Cancer 127: 138-147, 2010.

19. Hinz S, Weikert S, Magheli A, et al: Expression profile of the polycomb group protein enhancer of Zeste homologue 2 and its prognostic relevance in renal cell carcinoma. J Urol 182: 2920-2925, 2009.

20. Ntziachristos $\mathrm{P}$, Tsirigos A, Van Vlierberghe $\mathrm{P}$, et al: Genetic inactivation of the polycomb repressive complex 2 in $\mathrm{T}$ cell acute lymphoblastic leukemia. Nat Med 18: 298-301, 2012.

21. Hou T, Liang D, Xu L, et al: Atypical chemokine receptors predict lymph node metastasis and prognosis in patients with cervical squamous cell cancer. Gynecol Oncol 130: 181-187, 2013.

22. Shibata D: Mutation and epigenetic molecular clocks in cancer. Carcinogenesis 32: 123-128, 2011.
23. Valk-Lingbeek ME, Bruggeman SW and van Lohuizen M: Stem cells and cancer; the polycomb connection. Cell 118: 409-418, 2004

24. Zhou J, Roh JW, Bandyopadhyay S, et al: Overexpression of enhancer of zeste homolog 2 (EZH2) and focal adhesion kinase (FAK) in high grade endometrial carcinoma. Gynecol Oncol 128: 344-348, 2013.

25. Li H, Bitler BG, Vathipadiekal V, et al: ALDH1A1 is a novel EZH2 target gene in epithelial ovarian cancer identified by genome-wide approaches. Cancer Prev Res (Phila) 5: 484-491, 2012.

26. Chen SQ, Zhang HM, Li JB, et al: Analyzing simultaneous positive expression of EZH2 and P53 protein to improve predictive value in cervical squamous cell carcinoma. Int J Gynecol Cancer 24: 1653-1658, 2014.

27. Fang J, Zhang M and Li Q: Enhancer of zeste homolog 2 expression is associated with tumor cell proliferation and invasion in cervical cancer. Am J Med Sci 342: 198-204, 2011.

28. Breuer RH, Snijders PJ, Smit EF, et al: Increased expression of the EZH2 polycomb group gene in BMI-1-positive neoplastic cells during bronchial carcinogenesis. Neoplasia 6: 736-743, 2004. 